\section{Diagnosis and impact of disconnected pancreatic duct syndrome on endoscopic management of pancreatic fluid collections}

\begin{abstract}
Bang JY, Wilcox CM, Navaneethan U, Hasan MK, Peter S, Christein J, et al. Impact of disconnected pancreatic duct syndrome on the endoscopic management of pancreatic fluid collections. Ann Surg 2016. [Epub ahead of print].
\end{abstract}

This study reports findings of a retrospective evaluation of a database of patients from two large tertiary care centers in the USA with extensive experience in pancreatic endotherapy undergoing endoscopic drainage for pancreatic fluid collections (PFCs) from 2003 to 2015. The patients underwent an initial endoscopic/endoscopic ultrasound-guided (EUS) drainage, and if the response was deemed inadequate, further intervention in the form of multi-gate technique, dual modality drainage, or percutaneous necrosectomy was undertaken for clinical success. The authors report about the presence of disconnected pancreatic duct syndrome (DPDS) and its implication on outcome vis-à-vis success of intervention, requirement of hybrid methods or repeated interventions, length of hospitalization, requirement for surgery, etc. The diagnosis of DPDS was based on magnetic resonance cholangiopancreatography (MRCP) (eighty patients) or endoscopic retrograde cholangiopancreatography (ERCP) (180 patients) or EUS-guided pancreatogram (four patients) findings. Any patient requiring more than six endoscopic procedures was deemed to have failed treatment. While till 2008 no transmural stents were left, after 2008, transmural stents were left in situ to allow for the drainage of disconnected segment of the pancreas.

In a cohort of 361 patients, seventy patients could not be assessed for the presence of DPDS due to various reasons. Of the rest of 291 patients, 167 patients had DPDS while 124 had the continuity of the MPD maintained. The patients with DPDS were older than the other group and had a larger number of male patients with walled-off pancreatic necrosis (WON)-type collections which were larger or multiple in numbers. The drainage in DPDS patients was more likely to be trans-duodenal and required placement of a larger number of stents. These patients also needed enteral feeding tube placement more frequently because of a poor tolerance to orally administered feeds. Overall, $88 \%$ of patients achieved treatment success and these rates were similar in patients with or without DPDS. However, the presence of DPDS was associated with more requirements of additional (hybrid) procedures which were needed in one-third of the patients in contrast to being needed in only $4.8 \%$ of patients without DPDS. Endoscopic re-interventions were needed more frequently in patients who had an underlying DPDS vis-à-vis those who did not have DPDS (30\% and 18.5\%, respectively). Moreover, the need for surgical intervention for rescue of failed endoscopic therapy was also significantly higher in patients with DPDS as compared to patients without DPDS (13.2 vs. 4.8\%, respectively). The median duration of hospitalization was also longer in the patients with DPDS by a day (3 vs. 2 days). However, the recurrence of PFCs was not different among the two groups. However, among the patients with DPDS, the recurrence rates were significantly lower for patients with permanent indwelling transmural stents. Other than the presence of DPDS, presence of WON and collections more than $10 \mathrm{~cm}$ was also associated with the need for hybrid procedures. The authors concluded that DPDS significantly impacts the endoscopic management of PFCs with requirement of more complex and frequent endoscopic interventions with higher failure rates.

Bang JY, Navaneethan U, Hasan MK, Hawes RH, Varadarajulu S. EUS correlates of disconnected pancreatic duct syndrome in walled-off necrosis. Endosc Int Open 2016;4:E883-9.

Traditionally, the diagnosis of DPDS is based on ERCP or MRCP findings, demonstrating the disruption of pancreatic duct. The utility of EUS in this setting is uncertain. In this prospective observational study, the authors studied the EUS findings in 21 patients with WON who were undergoing EUS-guided drainage. The EUS findings were correlated with computed tomography (CT), MRCP, or pathological findings on the surgical specimen. The authors hypothesized that the diagnosis of DPDS can be established while doing EUS-guided drainage of the WON, thereby precluding the necessity of subsequent ERCP or MRCP. The diagnosis of DPDS by EUS was suggested by the presence of a well-defined PFC along the course of the main pancreatic duct and upstream pancreatic parenchyma and duct terminating into this PFC. The diagnosis of DPDS was confirmed after resolution of the WON by obtaining pancreatogram by the use of ERCP or EUS.

Of the 42 patients assessed, 21 patients were excluded from the study for various reasons including the lack of adequate visualization of the pancreatic duct or upstream gland. Of 
the included 21 patients, 15 were males, and gallstones and alcohol were the predominant etiology. Predominantly, the PFCs were located in the body-tail region of the pancreas. All the 21 patients demonstrated the termination of the upstream pancreatic parenchyma and the duct into the WON, thereby suggesting the presence of DPDS.

On follow-up, clinically, 20 patients resolved with endoscopic therapy while one needed surgical rescue. In these 20 patients, pancreatogram confirmed the presence of complete ductal disruption. The disruption was also demonstrated after examination of the resected specimen of the patient who underwent surgery. The authors concluded that there was $100 \%$ correlation of EUS findings for the diagnosis of DPDS with follow-up CT, pancreatography or surgical findings, and early diagnosis of DPDS while doing EUS-guided drainage which may have significant clinical implications.

\section{Commentary}

In recent times, endoscopic or EUS-guided transmural drainage of PFCs has changed the management strategy of complications related to acute and chronic pancreatitis. The minimally invasive nature of endoscopic interventions with advantages of lower cost and shorter duration of hospital stay has decreased the need for surgical intervention. ${ }^{[1]}$ DPDS is a condition which can be seen in patients with acute or chronic pancreatitis and results from damage to pancreatic duct causing a complete separation of upstream and downstream parts of the ducts. This results in the separation of a viable part of pancreatic parenchyma from the downstream duct and thereby the pancreatic secretions from the upstream part now tend to drain into the pancreatic collections or peritoneum (ascites) or externally (external pancreatic fistula). ${ }^{[2]}$ The causes of DPDS may include pancreatic trauma, surgery, malignancy, and acute or chronic pancreatitis. ${ }^{[2]}$ The usual reported site of pancreatic duct disruption is head and neck/body region, possibly related to the propensity of this region to ischemic damage. ${ }^{[3]}$ Transmural drainage of the PFC/WON, while creating the conduit of drainage of the pancreatic fluid into the gastrointestinal (GI) lumen, does not treat the pancreatic duct disruption. If, in the settings of DPDS, the transmural stents are removed, there are high chances of recurrence of PFCs. Therefore, the currently accepted strategy is to leave plastic transmural stents indefinitely to maintain the drainage into the GI lumen. ${ }^{[2,4,5]}$ Interestingly, not all patients with DPDS who have been successfully treated with transmural drainage develop recurrence of PFCs after removal of transmural stents. In one retrospective report, the occurrence of early stent migration, disruption in head, absence of endocrine and exocrine dysfunction, or pancreatic atrophy was associated with the recurrence of PFCs. ${ }^{[6]}$ Furthermore, leaving a single transmural stent was as effective as leaving two transmural plastic stents for avoiding the recurrence of PFC. ${ }^{[7]}$
With the advent of endoscopic/EUS-guided metallic stent placement for drainage of PFCs, it becomes more imperative that presence of DPDS is established. ${ }^{[1,8]}$ Since metallic stents cannot be left indefinitely, the endoscopist may want to replace these with plastic stents in patients with DPDS. Therefore, identification of at least a subset of these patients at the time of EUS-guided drainage may guide further therapy in such patients. A recently published study has suggested that DPDS can be classified into three groups: concurrent DPDS where severe necrotizing pancreatitis may disrupt the pancreatic duct in the early phase, delayed DPDS where the patients with acute pancreatitis develop a late onset/recognition of disruption, and chronic pancreatitis-related DPDS which is related to chronic pancreatitis. While the concurrent DPDS may be associated with complex fluid collection which may form WON, the other two variants are associated with the formation of pancreatic pseudocysts. ${ }^{[9]}$

The presence of DPDS identifies those patients who need multiple interventions and higher surgical intervention. Therefore, these recently reported studies would help improve the management and prognostication of patients with DPDS. However, the use of EUS has its limitations. The presence of a nondilated duct may impair visualization and assessment for the presence of DPDS. In addition, large collections may impair the correct visualization of MPD-WON relationship. Indeed, half of the patients in the reported study could not be assessed on EUS for the presence or absence of DPDS. To conclude, patients with DPDS constitute a more difficult-to-treat subgroup, and in a subset of patients, the disconnected duct may be identified using EUS.

Vishal Sharma, Surinder Singh Rana

Department of Gastroenterology, Postgraduate Institute of Medical Education and Research, Chandigarh, India

Address for correspondence: Dr. Surinder Singh Rana, Department of Gastroenterology, Postgraduate Institute of Medical Education and Research, Chandigarh - 160 012, India. E-mail: drsurinderrana@yahoo.co.in

\section{References}

1. Sharma V, Rana SS, Bhasin DK. Endoscopic ultrasound guided interventional procedures. World J Gastrointest Endosc 2015;7:628-42.

2. Varadarajulu S, Rana SS, Bhasin DK. Endoscopic therapy for pancreatic duct leaks and disruptions. Gastrointest Endosc Clin N Am 2013;23:863-92.

3. Pelaez-Luna M, Vege SS, Petersen BT, Chari ST, Clain JE, Levy MJ, et al. Disconnected pancreatic duct syndrome in severe acute pancreatitis: Clinical and imaging characteristics and outcomes in a cohort of 31 cases. Gastrointest Endosc 2008;68:91-7.

4. Rana SS, Bhasin DK, Rao C, Sharma R, Gupta R. Consequences of long term indwelling transmural stents in patients with walled off pancreatic necrosis and disconnected pancreatic duct syndrome. Pancreatology 2013;13:486-90. 
5. Téllez-Ávila FI, Casasola-Sánchez LE, Ramírez-Luna MÁ, Saúl Á, Murcio-Pérez E, Chan C, et al. Permanent indwelling transmural stents for endoscopic treatment of patients with disconnected pancreatic duct syndrome: Long-term results. J Clin Gastroenterol 2016. [Epub ahead of print].

6. Rana SS, Bhasin DK, Sharma R, Gupta R. Factors determining recurrence of fluid collections following migration of intended long term transmural stents in patients with walled off pancreatic necrosis and disconnected pancreatic duct syndrome. Endosc Ultrasound 2015;4:208-12.

7. Rana SS, Sharma R, Sharma V, Chhabra P, Gupta R, Bhasin DK. Prevention of recurrence of fluid collections in walled off pancreatic necrosis and disconnected pancreatic duct syndrome: Comparative study of one versus two long term transmural stents. Pancreatology 2016;16:687-8.

8. Ang TL, Kongkam P, Kwek AB, Orkoonsawat P, Rerknimitr R, Fock KM. A two-center comparative study of plastic and lumen-apposing large diameter self-expandable metallic stents in endoscopic ultrasound-guided drainage of pancreatic fluid collections. Endosc Ultrasound 2016;5:320-7.

9. Fischer TD, Gutman DS, Hughes SJ, Trevino JG, Behrns KE. Disconnected pancreatic duct syndrome: disease classification and management strategies. J Am Coll Surg 2014;219:704-12.
This is an open access article distributed under the terms of the Creative Commons Attribution-NonCommercial-ShareAlike 3.0 License, which allows others to remix, tweak, and build upon the work non-commercially, as long as the author is credited and the new creations are licensed under the identical terms.

\begin{tabular}{|l|c|}
\hline \multicolumn{3}{|c|}{ Access this article online } \\
\hline \multirow{2}{*}{ Website: } & Quick Response Code \\
www.jdeonline.in & \\
& DOI: \\
10.4103/jde.JDE_7_17 &
\end{tabular}

How to cite this article: Sharma V, Rana SS. Diagnosis and impact of disconnected pancreatic duct syndrome on endoscopic management of pancreatic fluid collections. J Dig Endosc 2017;8:46-8. 